\title{
The mass of Himalia from the perturbations on other satellites
}

\author{
N. V. Emelyanov ${ }^{1,2}$ \\ 1 Sternberg astronomical institute, 13 Universitetskij prospect, 119992 Moscow, Russia \\ e-mail: emelia@sai.msu.ru \\ 2 Institut de mécanique céleste et de calcul des éphémérides - Observatoire de Paris, UMR 8028 du CNRS, \\ 77 avenue Denfert-Rochereau, 75014 Paris, France
}

Received 20 May 2005 / Accepted 12 June 2005

\begin{abstract}
We made an attempt to determine the masses of two Jupiter's outer satellites, Himalia (J6) and Pasiphae (J8), on the basis of their perturbations on other satellites. For this, all observations available in the database of the Natural Satellite Data Center (NSDC, http://lnfm1. sai .msu.ru/neb/nss/index.htm) were used. New value of the product of Himalia's mass by the gravity constant $G m=(0.28 \pm 0.04) \mathrm{km}^{3} / \mathrm{s}^{2}$ was obtained (the uncertainty of one-sigma is given). We demonstrated that the determination of the mass is possible only because of one close approach of Himalia (J6) and Elara (J7) which took place on July 15th, 1949, when the distance between them was $65031 \mathrm{~km}$. Independent determination of Himalia's mass allowed to obtain the relationship between assumed values of density and geometric albedo of this satellite. Researches based on simulated observations showed that such a determination of the mass for the satellite Pasiphae (J8) is impossible.
\end{abstract}

Key words. ephemerides - planets and satellites: general - planets and satellites: individual: Jupiter

\section{Introduction}

To solve the problem of the origin and evolution of the Solar System, we need to know the physical parameters of the planets and satellites as well as properties of their orbital motion.

Since the outer planetary satellites are very small celestial bodies, only their stellar magnitudes may be determined from ground-based photometric observations. Masses of the outer satellites, their dimensions and albedos were unknown until recently. Approximate evaluations of masses and dimensions had been made based on hypothetical values of densities and albedos.

The physical properties of the irregular satellites is a very topical issue, given the recent surge of new discoveries. Only the mass of one other irregular satellite, S9 Phoebe at Saturn, has until now been determined from the Cassini spacecraft flyby in June 2004 (Jacobson 2004).

Himalia's mass in particular has a direct bearing on recent work by Christou (2005) who demonstrated (a) the ability of Himalia to scatter its satellite group significantly over the age of the solar system (b) that the magnitude of this scattering is critically dependent on Himalia's mass.

Researchers have an independent method of determination of a planetary satellite's mass which uses observations of other satellites whose motion is influenced by their mutual interactions. Such general approach was applied also to the determination of asteroid masses through their effect on other asteroids during close encounters (Michalak 2001). The possibility to determine the masses in this way depends on the interrelation between the accuracy of observations and the magnitude of mutual perturbations.

In this paper we made an attempt of a new determination of the masses of some outer Jovian satellites through the gravitational perturbations that they make.

\section{Orbital properties of the outer Jovian satellites}

By now, 54 outer satellites of Jupiter have been discovered which may be divided into two groups depending on the mean distances from the planet. The first group is represented by the satellites with semi-major axes in the range of 10-13 million kilometers with a prograde orbital motion. The satellites of the second group have semi-major axes between 20 and 25 million kilometers with a retrograde orbital motion.

We excluded from our consideration the satellites discovered in the last 6 years because, for the determination of the masses of satellites on the basis of their mutual perturbations, we should have a larger number of observations made during longer time intervals. Observations of these satellites cover only small portions of their orbits. Because of that, we separately considered two satellite groups: the first included Himalia (J6), Elara (J7), Lysithea (J10) and Leda (J13), while the second group included Pasiphae (J8), Sinope (J9), Carme (J11) and Ananke (J12). According to some evaluations based upon some photometry, the most massive satellite of the first group is Himalia (J6), while Pasiphae (J8) has the biggest mass among the satellites of the second group. We have set a task to determine the masses of the most massive satellite of each group using observations of other satellites. 


\section{Researches based on simulated observations}

Observations of these satellites made after their discovery up to now have a various accuracy and are distributed very unevenly in time. So, at first, we investigated the possibility to solve the problem under ideal circumstances using simulated observations. At the same time, evaluations of the accuracy of the determination of the mass of the satellite were made.

The motion of the satellites was simulated using numerical integration of the equations of motion. As initial conditions may be obtained only from observations, initial conditions of motion of the perturbed satellites and the mass of the perturbing satellite were improved concurrently. To define the parameters, we used the least squares fit which also gives the evaluation of the accuracy of the defined parameters.

To solve the problem, methods and calculating programs used were described by Emelyanov (2005). The program was expanded by the possibility to take into account the mutual interactions among satellites and to improve the perturbing mass of one of the satellites.

Simulated observations were produced using ephemerides calculation program which is also described in Emelyanov (2005). The program generates the values of geocentric right ascensions and declinations of an outer Jovian satellite in the interval 1905-2003 with a step of 90 days. To improve the mass of Himalia (J6), observations of Elara (J7), Lysithea (J10) and Leda (J13) were simulated. To improve the mass of Pasiphae (J8), we simulated observations of Sinope (J9), Carme (J11) and Ananke (J12). We took those initial conditions of integration that were found from observations (Emelyanov 2005). The masses of the perturbing satellites were taken to be $0.45 \mathrm{~km}^{3} / \mathrm{s}^{2}$ for Himalia (J6) and $0.013 \mathrm{~km}^{3} / \mathrm{s}^{2}$ for Pasiphae (J8). Other satellites were assumed to be massless. Values of simulated observed parameters were supplemented by random errors using random number generator. Any value of root-mean-square error could be assigned to the simulated observations.

Using the simulated observations, the initial conditions of integration of equations of satellite motion and the masses of the perturbing bodies were concurrently improved. Initial values of the masses of the perturbing satellites were set to zero. It was found that, after improvement, the masses differed from their initial simulated values by no more than the errors obtained using the least squares fit. We were most interested in obtaining the relationship between the errors of the simulated observations and the errors on the mass determination.

Let $\sigma_{G m}$ be the error of the product of the perturbing mass $m$ by the gravity constant $G$. This value is obtained after data are processed using the least squares fit. Let $\sigma_{d}$ be the root-meansquare value of angular distance between calculated and observed satellite positions, i.e. the error of observations which, in this case, was simulated by us. In determining the mass of Himalia (J6) the errors turned out to have the following relationship:

$\sigma_{G m}=0.031 \sigma_{d}$

where $\sigma_{G m}$ is expressed in $\mathrm{km}^{3} / \mathrm{s}^{2}$ and $\sigma_{d}$ is in arcseconds. It follows from this relationship that if, for example, the observations have accuracy of $0.2^{\prime \prime}$, then $G m$ of Himalia (J6) may be determined with accuracy of $0.0062 \mathrm{~km}^{3} / \mathrm{s}^{2}$ which represents $1.4 \%$ of its supposed value.

For Pasiphae (J8), the following relationship was obtained:

$\sigma_{G m}=0.32 \sigma_{d}$

which means that if the error of observations is $0.2^{\prime \prime}$, the error of $G m$ determination for Pasiphae (J8) is $0.064 \mathrm{~km}^{3} / \mathrm{s}^{2}$. This error is five times greater than the supposed value of $\mathrm{Gm}$.

Investigations based on simulated observations represent the ideal case of the problem. When real observations are used, the errors of the satellite mass determination may significantly rise. From this, taking into account that the best accuracy of present-day observations of the outer Jovian satellites is $0.2^{\prime \prime}$ (Emelyanov 2005), we conclude that the improvement of the mass of Pasiphae (J8) is impossible.

As regarding the mass of Himalia (J6), the estimation of errors gives hope that it can be improved using real observations.

In improving the parameters, the contribution of different sets of observations into the determination of the perturbing satellite's mass was investigated. For each observation we calculated the value of so-called sensitivity function:

$\Phi(t)=\sqrt{\left(\frac{\partial \alpha}{\partial m}\right)^{2}+\left(\frac{\partial \delta}{\partial m}\right)^{2}}$,

where $t$ is the date of observation, $\alpha, \delta$ are the geocentric right ascension and declination of the perturbed satellite, $m$ is the mass of the perturbing satellite.

Large values of the sensitivity function result in lesser errors in determination of satellite mass. Progressive growth of $\Phi(t)$ in time takes place only after approach of the perturbing and perturbed satellites in space. Such approaches turned out to be very rare. Since their discovery, there were only two occasions when Himalia (J6) and Elara (J7) approached one another at a distance less than 1 million kilometers: on September 18th, 1945, at a distance of $923701 \mathrm{~km}$ and on July $15 \mathrm{th}, 1949$, when the distance was $65031 \mathrm{~km}$. The latter approach was very close since the mean distances of the satellites from Jupiter are about 10-13 millions kilometers. After its discovery in 1938, Lysithea (J10) had two close approaches with Himalia (J6): on July 9th, 1954, when the mutual distance was $454216 \mathrm{~km}$ and on November 19th, 1954, when the mutual distance was $168891 \mathrm{~km}$.

The following feature of the $\Phi(t)$ function was discovered: for it to rise after the satellites' approach, it is necessary to integrate the partial derivatives at large time interval before the approach.

In order to determinate the mass of the perturbing satellite, it is necessary to have numerous observations of the perturbed satellite on the same interval of time as well before the close approach as after.

Researches also have shown that using the observations of Leda (J13) does not reduce the errors of determination of Himalia's mass. The mass may be determined with the same precision using only observations of Elara (J7) and Lysithea (J10). 
Table 1. The results of determination of $G m$ of Himalia (J6) for different sets of observations ( $N \mathrm{~J} 7$ - total number of used observations of Elara, $N^{\prime} \mathrm{J} 7$ - number of used observations of Elara made before 1949, $N \mathrm{~J} 10$ - number of used observations of Lysithea).

\begin{tabular}{lccccc}
\hline \hline$N$ & $N^{\prime}$ & $N$ & $\sigma_{d}$ & $\begin{array}{c}\sigma_{G m} \\
\left(\mathrm{~km}^{3} / \mathrm{s}^{2}\right)\end{array}$ & $\begin{array}{c}G m \\
\left(\mathrm{~km}^{3} / \mathrm{s}^{2}\right)\end{array}$ \\
$\mathrm{J} 7$ & $\mathrm{~J} 7$ & $\mathrm{~J} 10$ & $\left({ }^{\prime \prime}\right)$ & 0.270 \\
326 & 38 & 143 & 0.564 & 0.039 & 0.310 \\
326 & 38 & - & 0.551 & 0.041 & 0.310 \\
315 & 36 & 136 & 0.529 & 0.038 & 0.278 \\
315 & 36 & - & 0.521 & 0.039 & 0.297 \\
280 & 24 & 120 & 0.454 & 0.037 & 0.278 \\
280 & 24 & - & 0.442 & 0.039 & 0.284 \\
\hline
\end{tabular}

\section{Improvement of the mass of Himalia}

To improve the mass of Himalia (J6), observations of Elara (J7) and Lysithea (J10) were taken from the on-line NSDC database. This is the same set of observations which was used by the author (Emelyanov 2005) to construct the ephemerides of these satellites. The initial set of the observations contained 449 observed positions of Elara (119 of them were made before 1949) and 173 positions of Lysithea.

We excluded from the results of observations some systematic errors which were mentioned by Jacobson (2000) and in our paper (Emelyanov 2005). In addition, some evidently crude observations were excluded. But real observations do inevitably have some systematic errors which were not taken into account. Observations have different accuracies. Under these circumstances, one may expect that the determined mass will depend on the sets of the observations used. So we improved the mass in different ways which differ in the criteria defining the rejection of the most erroneous observations. The case when only observations of Elara (J7) are used was also considered. The results of determination of Himalia's mass for different sets of observations are given in Table 1.

The quality of solution obtained using least squares fit is characterized by the maximum coefficient of correlation $\max \left\{K_{\text {corr }}\right\}$ between the errors of the unknown mass and those of other defined parameters. In our solutions, when only observations of Elara (J7) were used, the maximum coefficient of correlation $\max \left\{K_{\text {corr }}\right\}$ was equal to 0.382 (for the correlation between the unknown mass and the initial coordinate $y_{0}$ of the perturbed satellite).

These values of coefficients of correlation indicate weak dependence of errors of the perturbing satellite's mass on the errors of other defined parameters. When observations of both Elara (J7) and Lysithea $(\mathrm{J} 10)$ were used, $\max \left\{K_{\text {corr }}\right\}$ ranged from 0.820 to 0.886 . The increasing of the coefficient of correlation in these cases may be explained by the fact that the observations of Lysithea (J10) began only in 1938. Attempts to improve the mass of the perturbing satellite using only observations of Lysithea (J10) end in failure - the error of the value is several times greater than the value itself.

To confirm the find of the most probable value of the mass of Himalia an additional analysis was made. The initial conditions of motion of Elara were obtained from observations using

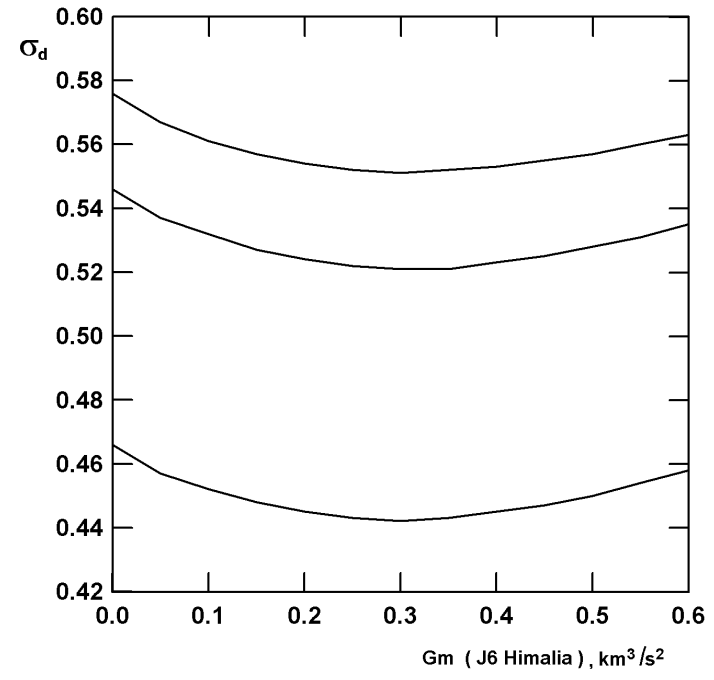

Fig. 1. The rms of angular distance between calculated and observed satellite positions $\sigma_{d}$ after the fit of the initial conditions of Elara versus the mass supposed for Himalia.

the least squares fit for a set of values of the mass of Himalia. The rms of angular distance between calculated and observed satellite positions $\sigma_{d}$ after the fit versus the mass supposed for Himalia are shown in Fig. 1.

In result, we may conclude that the most probable value of $G m$ of Himalia (J6) is $(0.28 \pm 0.04) \mathrm{km}^{3} / \mathrm{s}^{2}$.

\section{Comparison with the previous values of Himalia's mass}

Data on the parameters of Himalia (J6) were found in the following papers. Rettig (2002) defined from observations absolute magnitude of the satellite. Having assumed that the value of albedo is 0.04 , the radius turned out to be $85 \mathrm{~km}$. Using this value of radius and assumed density of $2.6 \mathrm{~g} / \mathrm{cm}^{3}$, Himalia's mass was calculated at Solar System Dynamics Group of the Jet Propulsion Laboratory (SSD JPL, http://ssd.jpl.nasa.gov). The value of mass multiplied by the gravity constant $G$ was found to be $0.45 \mathrm{~km}^{3} / \mathrm{s}^{2}$. From the satellite's absolute magnitude observed and assumed albedo of 0.03, Luu (1991) calculated a radius of $92 \mathrm{~km}$. During the Cassini spacecraft flyby of the Jupiter system, J6 Himalia was imaged from a range of $4.4 \times 10^{6} \mathrm{~km}$, exhibiting a twodimensional profile measuring $75 \times 60 \mathrm{~km}$ acros and inferred albedo of 0.05 (Porco et al. 2003).

Suppose that the satellite has the spherical shape. Let us introduce the parameter

$M=p \pi r^{2}$

where $p$ is the geometric albedo of the satellite, $r$-its radius. $M$ characterizes the satellite's absolute brightness and is obtained directly from observations. From the results of Rettig (2002) we have $M=907.92 \mathrm{~km}^{2}$. The results of Porco et al. (2003) give that $M=705.13 \mathrm{~km}^{2}$. 


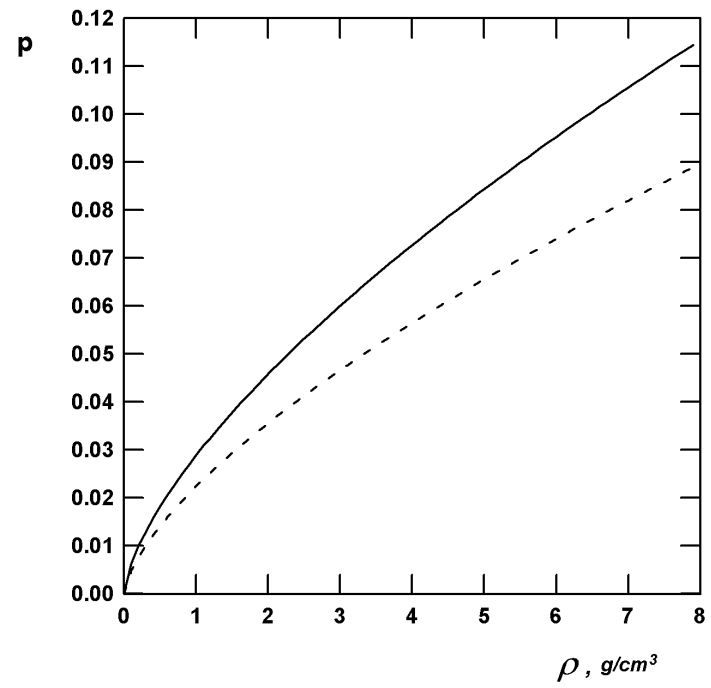

Fig. 2. The dependence of the possible values of albedo $p$ on density $\rho$ for $G m=0.28 \mathrm{~km}^{3} / \mathrm{s}^{2}$. Continuous line - the photometry from (Rettig 2002), dotted line - the photometry from Porco et al. (2003).

Let $\rho$ be the satellite's density. Then the last relationship may be written as having the form

$p \pi^{\frac{1}{3}}\left(\frac{3 m}{4 \rho}\right)^{\frac{2}{3}}=M$

from where we may get the relation between the values of $\rho$ and $p$

$\rho^{2}=K p^{3}$.

The coefficient $K$ depends on the results of the satellite's photometry and its adopted mass. For the mass of Himalia obtained in this research, using the photometry results of Rettig (2002), we have: $K=41577 \mathrm{~g}^{2} / \mathrm{cm}^{6}$. The results of Porco et al. (2003) give that $K=88755 \mathrm{~g}^{2} / \mathrm{cm}^{6}$. The corresponding dependences of $p$ on $\rho$ are shown in Fig. 2.
Taking the value of Himalia's radius measured using the image made by the Cassini-Huygens mission, from the mass obtained in our research it follows that the density of the satellite is $(3.33 \pm 0.47) \mathrm{g} / \mathrm{cm}^{3}$. In reality, Himalia (J6) may not have spherical shape, so the values of density and the above relationships may be very rough.

\section{Conclusion}

As seen from the behavior of the sensitivity function, determination of Himalia's mass turned out to be possible only due to its close approach to the satellite Elara (J7) on July 15th, 1949, at the distance of $65031 \mathrm{~km}$. Weak dependence of the result on the composition of observational data allow to assume that the obtained value $G m=(0.28 \pm 0.04) \mathrm{km}^{3} / \mathrm{s}^{2}$ is close to the real one. As regards other outer Jovian satellites, their masses cannot be determined using this method.

Acknowledgements. This work was supported by the Russian Foundation for Basic Research, project No. 03-02-17441.

\section{References}

Christou, A. A. 2005, Icarus, 174, 215

Emelyanov, N. V. 2005, A\&A, 435, 1173

Jacobson, R. A. 2000, AJ, 120, 2679

Jacobson, R. A., Antreasian, P. G., Bordi, J. J., et al. 2004, A\&AS, DPS meeting, 36, 15.02

Luu, J. 1991, AJ, 102, 1213

Michalak, G. 2001, A\&A, 374, 703

Porco, C. C., West, R. A., McEven, A., et al. 2003, Science, 299, 1541

Rettig, T. W., \& Walsh, K. 2002, BAAS, 34, 861 